\title{
La construcción semántica de problemas sociales en la política pública. El caso de la política de vivienda en Chile
}

\author{
The semantic construction of social problems on public policy. The case of housing \\ policies in Chile
}

Gabriela Azocar

Centro de Investigación Núcleo Milenio Modelos de Crisis, Universidad Adolfo Ibáñez, Chile.

Departamento de Sociología, Universidad de Chile, Chile.

RESUMEN

El estudio de las políticas públicas ha sido abordado principalmente por corrientes técnicas y teóricas que han puesto especial atención en los resultados y en los procesos bajo las cuales estas se conforman. Esta investigación analiza cómo el Estado construye los problemas sociales que aborda a través de formulaciones semánticas que condensan orientaciones y significados provenientes de distintas esferas sociales. El enfoque se desarrolla a partir de la revisión del caso de la política de vivienda en Chile. Los resultados indican que es posible identificar cuatro periodos que representan cambios sustantivos en la construcción estatal del problema de la vivienda: desde la habitación a la vivienda urbana, desde la semántica del déficit a la juridificación de la vivienda, desde la juridificación a la semántica económica y, finalmente, a la semántica de la inclusión social por medio de la vivienda. La estrategia metodológica utilizada se basa en la revisión de leyes y decretos en los que se sedimentan y legitiman los procesos de toma de decisiones que dan forma a la política pública.

PAlabRAS ClavE: Semántica; Sistemas sociales, Políticas públicas; Vivienda; Legislación

\section{ABSTRACT}

The study of public policy has been addressed mainly from technical and theoretical perspectives that paid special attention to the results and processes under which they are designed. This paper analyzes how the State constructs its social problems on the basis of semantic formulations that condense orientations and meanings originated in multiple social spheres. The approach is developed through the analysis of the Chilean case of housing policy. Results indicate that four periods can be identified, each representing substantive changes in the State construction of the housing problems: from dwelling place to house, from the semantics of deficit to the juridification of housing, from juridification to the economic semantics, and finally, to the semantics of social inclusion through housing. The methodological strategy bases on the revision of acts and laws, which condenses and legitimates decision-making processes that shape public policy.

KEYWORDS: Semantics; Social systems; Public policy; Housing; Laws

\author{
REVISTA MAD - REVISTA DEL MAGÍSTER EN ANÁLISIS SISTÉMICO APLICADO A LA SOCIEDAD \\ ISSN 0718-0527 \\ Facultad de Ciencias Sociales, Departamento de Antropología. \\ Universidad de Chile \\ www.revistamad.uchile.cl
}

DOI: $10.5354 / 0718-0527.2016 .42795$ 


\section{INTRODUCCIÓN ${ }^{1}$}

La teoría del Estado durante el siglo XX ha desarrollado sistemáticamente una amplia reflexión en torno a la función de las políticas públicas en sociedades modernas y sus consecuencias para la inclusión social y para el ámbito más político de la legitimización de distintas formas de organización del Estado (Habermas 1973; Luhmann 1993; von Beyme 1994; Scharpf 1999; Rosanvallon 2000; Mayntz 2001; Rawls 2002; Parsons 2007; Willke 2014). En la mayoría de estas perspectivas, el énfasis está puesto, por un lado, en el proceso de construcción de políticas públicas como articulación de intereses individuales o coordinación de perspectivas de diversos actores corporativos y, por otro, en su capacidad para formar instituciones de inclusión social que logren procesar y dar respuesta a las demandas de los públicos, en un marco normativo que promueva el trato justo e igualitario de los miembros de una comunidad política.

Mediante el concepto de "coordinación social", la tradición sistémica ha contribuido al análisis de las políticas públicas, especialmente en el proceso de formación y articulación de ellas (Lechner 2003; Willke 2007; Mascareño 2010; Börzel \& Risse 2010; Schmidt 2013; Kjaer ,2014). Paralelamente, desde este enfoque se ha procurado esclarecer las condiciones en que se desarrollan procesos de inclusión social (Arnold 2012; Robles 2005; Cadenas 2016; Mascareño \& Carvajal 2015), así como también a la evaluación de resultados (Zeitlin et al. 2005; Vega 2006; Atkinson 2009; Ziccardi 2008). El presente artículo busca abrir una variante distinta pero complementaria en el análisis de políticas públicas desde una perspectiva sistémica. El enfoque se centra en la dimensión de formación de las políticas públicas, pero en vez de poner el énfasis en la coordinación de actores y organizaciones, el artículo se enfoca en la construcción semántica de problemas sociales que el Estado realiza, a la cual él mismo intenta dar respuesta por medio de legislación y políticas.

Las bases teóricas de este enfoque son las siguientes. Las necesidades y demandas de actores sociales son, en principio, externas a la política. Aplicando el proceso de comunicación de tres selecciones de Niklas Luhmann (1991) al caso analizado, los actores toman la posición del alter que ofrece determinados contenidos comunicativos y la política, a su vez, la posición del ego que comprende y debe reconstruir aquella oferta comunicativa internamente. Para lograr esto, la política tiene que crear un correlato interno de las comunicaciones de los actores concretos. Esta función la cumple el concepto de públicos (Luhmann 2009). Los públicos son, por tanto, una construcción propia del sistema político diseñada para situar en su interior la comprensión de demandas externas y ponerlas en relación con los otros elementos de su diferenciación interna: la administración y los partidos.

\footnotetext{
${ }^{1}$ El presente trabajo ha contado con el patrocinio del Núcleo Milenio Modelos de Crisis (NS 130017).
} 
Producto del proceso de comprensión que el sistema político realiza de las demandas de los públicos, el sistema desarrolla diversas formulaciones semánticas (Luhmann 2007; Stäheli 1997, Dockendorff 2007) que condensan la red significados por medio de los cuales esas demandas se transforman en problemas sociales que la política se propone resolver. En su operación interna, la política conecta esas semánticas con la dimensión de administración para la formulación de políticas públicas y, adicionalmente, las conecta con el proceso político-legislativo para su transformación en decisiones colectivamente vinculantes que quedan enmarcadas en leyes (Luhmann 2009).

De esta manera, las semánticas políticamente construidas, a partir de las comunicaciones de actores concretos, dan forma a los problemas sociales que la política pública, volcada en legislación, asigna a públicos internos y que busca resolver paralelamente por medio de sus propias operaciones. La semántica política juega, por tanto, un rol fundamental en la construcción de problemas sociales, para los cuales se diseñan política pública y legislación. Puesto que ese proceso operativo es constante en el tiempo, la construcción del problema está sometida a transformaciones más o menos profundas que dependen del mismo proceso comunicativo descrito. En tanto la relación entre alter y ego no acabe, el problema social de referencia al que la política pública se dirige tampoco deja de cambiar.

Sobre estas bases teóricas, la hipótesis de este artículo tiene una doble dimensión. Por una parte, la semántica política que da forma y contenido a la política pública y la legislación sobre vivienda es la que internamente construye el 'problema social de la vivienda' en Chile durante el siglo XX, y por otra, puesto que esta construcción se despliega en una dimensión temporal, el mismo problema social de la vivienda evoluciona en su composición semántica y en los rendimientos que la esfera política ofrece a los públicos. Metodológicamente, el análisis se construye sobre la legislación relativa a la vivienda durante el siglo XX. La información obtenida de las leyes y decretos analizados se considera evidencia del acoplamiento entre política y legislación, en el entendido que las leyes sedimentan y legitiman aquellos procesos de decisión vinculante que se plasman en las políticas públicas. En este contexto, la ley es observada en un triple sentido: a) indica las expectativas que el Estado tiene sobre sus propias operaciones en el ámbito de la vivienda; b) indica también las expectativas sobre las cuales el público construye sus aspiraciones; y c) señala las operaciones que el Estado se obliga a realizar en este ámbito. Mediante citas y referencias a distintos cuerpos legales, se reconstruirá la evolución de la política de vivienda.

Para desplegar este análisis, se analizarán las transformaciones semánticas sobre el problema de la vivienda en Chile entre 1906 (Ley de Habitaciones Obreras) y 1965 (creación de Ministerio Vivienda), en el que paulatinamente se distingue entre habitación y vivienda (1), se continúa 
con el período 1965 a 1978 (sistema de subsidio a la vivienda) donde la semántica dominante del problema es la del 'déficit' (2), para luego analizar el período 1978 a 1990 (aumento gasto público), en el que predomina una construcción de semánticas económicas (3) y el período 1990 a 2010 (redestinación de recursos) donde la semántica de la inclusión juega un rol central en la definición del problema (4). El trabajo concluye con una discusión de los hallazgos y una reflexión acerca de la importancia de la observación semántica de las políticas públicas y sus posibilidades de complementación con otros enfoques de política (5).

\section{DESDE LA HABITACIÓN A LA VIVIENDA URBANA (1906-1965)}

La promulgación de la Ley de Habitaciones Obreras el año 1906 ha sido considerada por distintos autores (Torres 1986; Ducci 1997; Hidalgo 1999; Simian 2010; Rivera 2012) como el hito que da cuenta de las primeras acciones estatales que abordan de manera concreta el problema de la vivienda en Chile. A partir de esta ley, diversas instituciones adoptan una serie de decisiones sobre la manera de intervenir este problema. Se trata de un periodo en el que el Estado reacciona ante las demandas de vivienda de manera dispersa, sin lograr diferenciar su intervención de otros ámbitos como el trabajo, la sanidad pública y el bienestar social. Esta forma caótica de operación es superada con la creación del Ministerio de la Vivienda y Urbanismo el año 1965, institución que centraliza la toma de decisiones sobre el problema de la vivienda, dando cuenta de una clara especialización del Estado en esta materia. Este hito determina el fin de este periodo de análisis.

El momento histórico en el que la Ley de Habitaciones Obreras es promulgada se caracteriza por los cambios sociales de principios del siglo XX, asociados principalmente al desarrollo creciente de las grandes ciudades y el proceso migratorio campo-ciudad. Una de las principales consecuencias del traslado de grandes masas de trabajadores a los principales centros urbanos del país fue la rápida densificación de ciudades como Valparaíso, Concepción, Antofagasta, Iquique, Talca Chillan y Santiago, lo que se produjo en base al asentamiento de esta población en condiciones de extrema precariedad (Geisse \& Valdivia 1978; Hidalgo 1999; Forrary 2007;). Las insalubres condiciones de vida de los obreros urbanos que residían en conventillos de restringidas extensiones, repercutieron en un aumento progresivo de las tasas de mortalidad y de enfermedades infecciosas en el país. En este contexto es que se devela una primera configuración del problema de la vivienda en Chile, cuya referencia semántica se focaliza principalmente en la noción de salud pública:

Si fuere calificada inhabitable por vetustez, existencia en ella de una infección permanente capaz de dañar a sus propios moradores o a los de las casas vecinas, u otra causa que impida la reparación en términos convenientes para la salud, se pasará la misma comunicación prescrita en el inciso precedente, con expresión 
de la causa que le da este carácter (Artículo Nº10. Ley Nº1838 de los Consejos de Habitaciones. Ministerio del Interior, 20 de febrero de 1906)

Si bien esta primera aproximación a la definición del problema de la vivienda da cuenta de un agudo dilema social, es a su vez una forma simple, concreta y focalizada de comprensión del problema por el que se reclama intervención estatal. El Estado responde a esta demanda a través de políticas públicas que persiguen el saneamiento de los espacios de morada con el fin de superar los problemas de salud y hacinamiento generados en los conventillos o habitaciones colectivas. 'Saneamiento', 'salubridad' e 'higienización' son los conceptos que predominan en las decisiones contenidas en las primeras normativas generadas para mejorar las condiciones de vida de los obreros urbanos. A partir de estas referencias semánticas, se delimita un ámbito particular de intervención estatal, a la vez que se determina el curso a seguir de los procesos venideros de toma de decisiones.

Serán declaradas insalubre o inhabitables las casas destinadas a darse en arrendamiento cuyas habitaciones no reúnan las condiciones que exija la vida, bajo el punto de vista de la distribución de las piezas, su nivel con relación a los patios i [sic] calles, el cubo de aire, la luz, la ventilación i [sic] demás preceptos de la hijiene [sic] (Artículo No8. Ley N¹838 de los Consejos de Habitaciones, Ministerio del Interior, 20 de febrero de 1906)

Las políticas que inicialmente se implementan para abordar el problema de las habitaciones obreras establecen requerimientos de ventilación, existencia de espacios de circulación y condiciones de higiene de las residencias de los obreros y sus familias. Esto da paso a la definición de regulaciones sobre las condiciones materiales que debe cumplir una vivienda desde una perspectiva técnica, orientación que permanecerá presente durante todo el desarrollo de la política de vivienda. La referencia semántica de la salud por el contrario irá perdiendo progresivamente su capacidad de influencia en la toma de decisiones a medida que esta dimensión del problema empieza a ser superada.

Durante las primeras décadas del siglo $X X$ se produce una primera evolución en la manera en que el Estado define las necesidades y demandas de los públicos. El foco de la intervención estatal se reorienta paulatinamente a la delimitación de las condiciones materiales de la vivienda y del entorno que le circunda. El Estado comienza a comprender que las demandas de los actores no se limitan solo a la necesidad de habitar un espacio y que el problema de la vivienda debe considerar las condiciones contextuales donde ésta se sitúa. En este marco, la definición de lo urbano adquiere particular influencia en la determinación del problema (MINVU 2007). La noción de habitabilidad se extiende fuera de los límites de los muros de la residencia y pasa a entenderse como un mecanismo de desarrollo social y 
vinculación con la comunidad. Se identifica entonces la necesidad de dotación de espacios comunes de circulación y recreación adecuados para el desarrollo de quienes viven conjuntamente en lo que se configura como los nuevos barrios urbanos.

Las poblaciones que se edifiquen en conformidad a la presente lei deberán contar cuando el Consejo Superior de Bienestar Social lo crea oportuno, con locales para todo o alguno de los siguientes servicios: escuelas, plaza de juegos infantiles, policlínico, farmacia, mercado, restaurant popular, teatro y correo. (Artículo $\mathrm{N}^{\circ} 25$. Decreto Ley $\mathrm{N}^{\circ} 308$ de Habitaciones Baratas. Ministerio de Higiene, Asistencia, Previsión Social y Trabajo, 9 de marzo de 1925)

Otro hito relevante que da cuenta de la manera en que el problema de la vivienda se transforma y ajusta a las características de los públicos, se produce a inicios de la década de 1930, cuando el concepto de "habitaciones obreras" es reemplazado por el de "habitación popular" (MINVU 2007). Esto da cuenta de un nuevo giro en la orientación de la política pública. En las normativas de estos años se define la habitación en función de las características materiales y del entorno de las viviendas destinadas a la población de menores recursos, lo que enfatiza la observación del problema desde la esfera económica. La 'habitación popular' adquiere un contenido simbólico: pasa a ser el término utilizado para denominar el lugar en el que reside la 'clase trabajadora', 'los obreros', 'las poblaciones', 'los sectores populares' y las 'personas de escasos recursos', todas denominaciones con las que se identifica a los principales afectados por el problema de vivienda en esta época. En este contexto, los públicos a los que el Estado dirige sus rendimientos se diferencian en función del precio del arriendo o el valor de las viviendas a las que pueden acceder los trabajadores, fijándose con ello las fronteras entre quienes son y no son considerados como objeto de la intervención estatal.

Se entenderá por Habitación Popular, para los efectos de esta ley, aquella cuya renta de arrendamiento mensual de las destinadas a este objeto, no exceda de $\$$ 200 y la casa unifamiliar cuyo precio no sea superior a \$20,000 en las ciudades de Santiago, Valparaíso, Antofagasta e Iquique. En las demás ciudades, el Presidente de la República determinará estos valores máximos los que no podrán exceder de los ya fijados (Artículo No3. Decreto con Fuerza de Ley N³3. Ministerio de Hacienda, 4 de abril de 1931)

Es importante señalar que, si bien el término 'habitación' figura en todas las normativas sobre la materia generadas entre 1906 y 1960, este va perdiendo predominancia en la medida que paulatinamente es reemplazado por el de 'vivienda', concepto que se estabiliza como denominación de la política pública a fines de este periodo. En la idea de vivienda se conjuga una concepción más amplia y compleja de un problema que no puede ser definido desde una única perspectiva. En ello es clave la creación de la CORVI en 1953 (Sabatini \& Soler 1995). 
Créase, mediante la fusión de la Caja de la Habitación y de la Corporación de Reconstrucción, una entidad autónoma de Derecho Público con personalidad jurídica, con el nombre de "Corporación de la Vivienda" dependiente en sus relaciones administrativas del Ministerio de Obras Públicas (Artículo Nº1, Decreto con fuerza de Ley $\mathrm{N}^{\circ} 285$. Ministerio de Hacienda, 5 de julio de 1953)

La CORVI representa el primer uso oficial del concepto de vivienda en un sentido más amplio que el de habitación, en tanto integra las distintas referencias semanticas y significados hasta ahora atribuidos a este problema. La vivienda es entendida como un requisito de subsistencia, como infraestructura material destinada a la morada y su entorno, como un mecanismo de vinculación con la comunidad y como una forma de diferenciación económica entre quienes cuentan o no con recursos monetarios para acceder a una residencia adecuada. Cabe señalar que la referencia económica adquiere especial relevancia en la comprensión del problema de la vivienda. Esto se observa en las leyes de la década de 1950, en las que el término vivienda se encuentra regularmente acompañado por calificativos como: 'económica', 'popular' y 'de bajo precio'.

Que es indispensable colocar la vivienda especialmente al alcance de las clases modestas del país, creando sistemas de adjudicaciones a plazos y financiamientos convenientes o de arrendamientos adecuados (Decreto con Fuerza de Ley $\mathrm{N}^{\circ} 285$. Ministerio de Hacienda, 5 de agosto de 1953)

Se considerarán "viviendas económicas", para los efectos del presente decreto con fuerza de ley, las que se construyan en conformidad a sus disposiciones, tengan una superficie edificada no superior a 140 metros cuadrados por unidad de vivienda y reúnan los requisitos, características y condiciones que determine el Reglamento Especial que dicte el Presidente de la República" (Artículo Nº1, Decreto con Fuerza de Ley N². Ministerio de Hacienda, 31 de julio de 1959)

El reemplazo del concepto de habitación por el de vivienda conjuga varias ideas: a) requisitos sanitarios; b) normas técnicas de construcción; c) el contexto urbano y comunitario en el que se inserta la vivienda; y d) distinciones económicas de los públicos a los que el Estado dirige sus rendimientos. La definición del problema se hace por tanto más compleja y policontextural ${ }^{2}$, lo cual repercute en la diversificación de las acciones estatales con las que éste es abordado. En síntesis, podemos decir que las políticas públicas de vivienda surgen como una decisión ante la amplia incertidumbre generada por la situación de marginalidad extrema en la que se desarrollaba la vida de los nuevos trabajadores urbanos que recientemente habían emigrado del campo a la ciudad. La dinámica decisional que da inicio a esta política pública, implica que la intervención del problema refiera a la carencia de

\footnotetext{
${ }^{2}$ Compuesto por varias dimensiones o estructuras.
} 
condiciones de salud, lo que se vincula directamente con escases de recursos económicos. A esto se suma la necesidad de considerar las condiciones materiales y el entorno urbano donde se inserta la vivienda. Esta diversidad semántica y operativa se mantiene y refuerza con la creación del Ministerio de la Vivienda y Urbanismo, hito que marca el inicio del siguiente periodo.

2. DeSDE LA SEMÁNTICA DEL DÉFICIT A LA JURIDIFICACIÓN DE LA VIVIENDA (1965-1978)

Este segundo periodo de análisis se inicia con la creación del Ministerio de la Vivienda y Urbanismo (en adelante MINVU) el año 1965 y culmina con la promulgación del sistema de subsidio a la vivienda (1978). Se caracteriza por el surgimiento de una institucionalidad pública especializada en el desarrollo e implementación de políticas públicas de vivienda. El MINVU será la institución estatal que estará encargada en lo sucesivo de la implementación de esta política. El periodo culmina el año 1978 cuando, en el contexto del régimen militar, se genera el sistema de subsidios a la vivienda.

Con la creación del MINVU, la noción de vivienda se consolida como denominación central en las normativas que establecen mecanismos de acceso diferentes tipos de inmuebles. A partir de esto, el problema de la vivienda es entendido como un problema específico y particular del cual el Estado debe encargarse, en tanto es éste quien asume la responsabilidad y control del conjunto de acciones que se desarrollan para su intervención. La creación de la CORVI en 1953, -pero particularmente la creación del MINVU en 1965- es un importante indicador de esta responsabilidad.

Durante este periodo calificativos tales como 'económica', 'social', 'básica' y 'progresiva' se utilizan con frecuencia para delimitar a quienes va dirigida la acción del Estado. Esto indica que la población de menores recursos económicos representa al público principal sobre el cual la política pública opera, pero se debe considerar que estos dejan de ser los destinatarios exclusivos de la acción estatal. A partir de la década de 1960, los sectores medios y en particular los funcionarios públicos se integran como nuevos beneficiarios de las políticas de Estado.

El Fisco, las instituciones semifiscales, las empresas fiscales y del Estado, los organismos de administración autónoma y, en general, todas las oficinas o funcionarios pagadores del sector público, estarán obligados a descontar de las remuneraciones y pensiones de sus respectivos funcionarios, empleados, obreros y pensionados, los dividendos a que se encuentren obligados por créditos hipotecarios de carácter habitacional. (Artículo N89, Decreto Nº121. MINVU, 19 de octubre de 1967)

A la vez que el problema de la vivienda adquiere mayor especificidad, empiezan a operar distinciones internas en el proceso de toma decisiones 
que configuran la política pública. Esto da cuenta del modo en que el problema integra en su definición distintas referencias a elementos territoriales, técnicos, económicos, políticos y legislativos, los que representan a su vez diferentes estrategias que el Estado implementa para su intervención.

Por un lado, la distinción entre vivienda urbana y rural surge de la necesidad de diferenciar las necesidades de estos tipos particulares de población, las que claramente se asocian a las condiciones territoriales en las que viven. Como se señala en la legislación de 1966:

La Corporación de Servicios Habitacionales está encargada de atender los problemas de vivienda de la población del país, preferentemente en sus niveles socio-económicos medio y bajo; desarrollar programas de asistencia técnica, social, económica, educacional y cultural, relacionados con el problema habitacional de los barrios, poblaciones y grupos humanos y, en general, realizar todas las actividades que digan relación con el bienestar habitacional de la población urbana y rural, dentro de los planes y programas elaborados por el Ministerio de la Vivienda y Urbanismo. (Artículo $\mathrm{N}^{\circ} 2$, Decreto $\mathrm{N}^{\circ} 485$. MINVU, 15 de septiembre de 1966)

Desde un punto de vista técnico-científico, el lenguaje utilizado en las normativas de vivienda también adquiere mayor especificidad. La noción de déficit que ya estaba presente hacia fines del periodo anterior, empieza a ser aplicada con regularidad, lo que tiene estrecha relación con que en este periodo se empiezan a utilizar de manera regular herramientas de investigación como el Censo de Vivienda que permiten estimar la magnitud del problema en términos de acceso/no acceso a la vivienda (Arellano 1982). El concepto de déficit, sin embargo, es utilizado no sólo como una referencia de la dimensión técnica del problema. El déficit pasa a ser el objetivo central de la intervención pública, aquello hacia lo que las decisiones del Estado se enfocan, en tanto debe ser superado, adquiriendo con ello ribetes económicos políticos y normativos. Las declaraciones del Ministro de Obras Públicas en el Gobierno del presidente chileno Eduardo Frei Montalva, con motivo de la creación del MINVU, son ilustrativas de este punto:

Estimamos que el Ministerio de Vivienda y Urbanismo será el órgano administrativo que hará posible la ejecución racional del Plan Popular de Viviendas, que constituyen la meta mínima que nos hemos propuesto alcanzar. Lograda ésta habremos obtenido la absorción del aumento vegetativo de la población, objeto hasta hoy no alcanzado por muy plausible y dignos de consideración que sean los esfuerzos realizados hasta la fecha. Y aun así no habremos empezado a disminuir el déficit de arrastre ya existente. (MINVU, 1964, p. 11).

La noción de déficit representa una sofisticación en la observación del problema, en tanto su estimación implica distinguir entre niveles y tipos de demandas. Esto se ve reflejado en que desde una perspectiva técnica, pero 
con connotaciones políticas y económicas, se empiezan a diferenciar distintos tipos de vivienda según su nivel de avance en la construcción, lo que está estrechamente ligado a las múltiples soluciones habitacionales que se diseñan como parte de esta política. Podemos diferenciar entre: a) viviendas 'económicas', 'básicas', 'sociales' y 'definitivas' como aquellas que se entregan al beneficiario, una vez su edificación ha sido completamente terminada; b) viviendas 'progresivas', 'mínimas' y 'autoconstruidas', cuando lo que se entrega son los cimientos básicos o los recursos para que los beneficiarios construyan o culminen la construcción de sus moradas; y c) viviendas de 'emergencia' y 'prefabricadas' como soluciones ante sucesos catastróficos tales como terremotos e inundaciones.

Confeccionar planos, tipos o modelos de "viviendas económicas", mínimas, progresivas, sociales, etc., y planos tipos del equipamiento comunitario que corresponda, con sus respectivas especificaciones y cálculos, con el fin de que se proporcionen en forma permanente a los interesados que lo soliciten, fijándoles precios accesibles a los grupos de más bajos ingresos. (Artículo $\mathrm{N}^{\circ} 23$, Decreto $\mathrm{N}^{\circ}$ 355. MINVU, 11 de marzo de 1977)

Estos diferentes niveles de construcción se asocian a distintos beneficiarios que se caracterizan según su capacidad adquisitiva o de endeudamiento. Las viviendas culminadas están destinadas a quienes tienen capacidad de ahorro y endeudamiento para su compra, los que corresponden principalmente a funcionarios públicos y familias de sectores medios-bajos. Aquellas que se entregan para la autoconstrucción están destinadas a los sectores de menores ingresos que no cuentan con un capital inicial que les permita acceder a una vivienda completamente construida. Esta última medida se relaciona con las políticas de erradicación de campamentos, pues durante este periodo la toma ilegal de terrenos se instala como una práctica frecuente en los sectores de mayores carencias económicas. La vivienda autoconstruida pasa a ser la solución que se ofrece los pobladores que se trasladan a vivir a zonas periféricas de la ciudad dotadas de un equipamiento urbano básico (Castillo 2010).

La creación del MINVU conllevó también a la introducción de nuevos conceptos en la denominación de la vivienda provenientes de la esfera del derecho. Ello se observa en una creciente juridificación de la nomenclatura asociada a la vivienda. Las nociones de 'inmueble' y 'domicilio' empezaron a ser utilizadas con mayor frecuencia cada vez que se establecían regulaciones sobre la adquisición legal de la vivienda, los mecanismos de endeudamiento para la compra de viviendas, su herencia y su expropiación.

Los inmuebles afectos a deudas que en virtud de esta ley dejen de ser reajustables o deban pactarse sin reajustabilidad, estarán sujetos a la prohibición de enajenar y arrendar por quince años, contados desde la fecha de la celebración del contrato que originó el crédito o saldo de precio. (Artículo N5, Ley N¹7663. MINVU, 30 de mayo de 1972) 
Las distinciones territoriales, técnico-científicas, políticas, económicas y jurídicas antes señaladas pueden ser entendidas como diferentes referencias simbólicas que confluyen en un espacio temático común definido por la política de vivienda. Se trata de diferentes perspectivas, desde las cuales se observa y comprende el problema de la vivienda en este periodo, y en función de las cuales se introducen distintas formas de operación en la intervención del mismo. Esto implica que existen denominaciones semánticas comunes que adquieren significados diversos según el uso y tipo de decisión que se adopte al momento de idear estrategias de intervención. Es posible hablar entonces de un espacio contextual particular en el que se interconectan formas de observar y operar de distintos sistemas parciales, el que se estructura a partir de la manera en que se define y comprende el problema de la vivienda. Esta diversidad semántica y operativa, sin embrago, se verá radicalmente restringida con la instauración del subsidio a la vivienda el año 1978. Con la promulgación de esta política, la referencia económica coloniza la definición del problema y los mecanismos de intervención estatal.

\section{EL PREDOMINIO DE LA ECONOMÍA EN LA SEMÁNTICA DE VIVIENDA (1978-1990)}

La promulgación del sistema de subsidio a la vivienda (1978) determina un cambio trascendente en el modo en que el problema es definido y en la forma de operar del Estado. La menor intervención estatal, en función de una mayor apertura a las leyes del mercado y el progresivo aumento de la participación del sector privado en la construcción de viviendas para sectores medios y altos, son los rasgos característicos de este periodo. Las medidas adoptadas para atender a los sectores de menores recursos fueron la entrega de subsidios y la erradicación a zonas periféricas de las grandes ciudades. Tales transformaciones implicaron una serie de adaptaciones y aprendizajes durante el proceso de implementación de esta nueva política, las que provocaron numerosas y sucesivas modificaciones a las leyes y ordenanzas con las que se implementaron estos cambios. El término de este periodo ésta delimitado por el retorno a la democracia el año 1990, hecho que da inicio a una serie de transformaciones en la política de vivienda. Estas persiguieron resolver los problemas generados por la desregulación de la política, el aumento del gasto público en esta materia y la incorporación de una mayor diversidad de intereses y expectativas en torno al acceso a la vivienda.

En este tercer periodo se suspende el proceso de creciente integración de referencias simbólicas y operaciones en torno al problema de la vivienda. Durante estos años, la referencia económica del problema pasa a predominar por sobre las de tipo técnico, urbana y jurídica, las que se supeditan a los requerimientos impuestos desde esta esfera. Esto se observa en que la 
definición del problema a intervenir pasa a centrarse en la noción de pobreza la que, en esta etapa, es comprendida como ausencia de recursos económicos. Esto implicó que el accionar del Estado se focalizó exclusivamente en la población "de bajos ingresos" y la población "marginal" (Rugiero 1998). La primera denominación alude a quienes poseen recursos suficientes, aunque escasos, para ahorrar y acceder a una deuda hipotecaria, mientras que la segunda se refiere a quienes carecen de estas posibilidades económicas de ahorro y endeudamiento. Esto implicó la exclusión explícita de todos los otros sectores de la población que contaban con ingresos estimados como suficientes para solucionar de forma independiente sus necesidades de vivienda en la esfera del mercado. Como lo señala la legislación con claridad hacia el fin de este período:

No podrán postular al subsidio correspondiente al tramo de valor de vivienda de hasta 400 Unidades de Fomento, aquellas personas cuya renta mensual, incluida la de su cónyuge, exceda, a la fecha de la postulación, del equivalente a 15 Unidades de Fomento. (Artículo N4, Decreto N44. MINVU, 9 de abril de 1988)

La posición de las personas en una estructura social diferenciada por estratos socioeconómicos pasa a ser el requisito primordial para identificar a quiénes, desde el Estado, son definidos como los afectados por el problema de la vivienda. La implementación de instrumentos tales como la Ficha de Caracterización Socioeconómica (CAS) reflejan cómo opera esta particular distinción.

Los SERVIU abrirán un registro en la respectiva región, en el cual inscribirán a los interesados, manteniendo en él la información requerida para efectuar las correspondientes selecciones. Dicha información, estará referida especialmente a la individualización y domicilio del postulante, a la individualización de su cónyuge y de sus hijos menores de dieciocho años, a su ubicación dentro del sistema de estratificación social, expresado en "Ficha CAS", y al ahorro que tuviere depositado el postulante o su cónyuge en una Cuenta de Ahorro a Plazo para la Vivienda. (Artículo $N^{\circ} 7$, Decreto $\mathrm{N}^{\circ} 62$. MINVU, 20 de junio de 1984)

Lo que se define como el 'interés social' del Estado refiere ahora a la atención de las necesidades de vivienda de la población que presenta mayores carencias económicas. La gran mayoría de las normativas generadas durante este periodo persiguen reglamentar la asignación de recursos públicos para la construcción de viviendas destinadas a esta población. A diferencia de lo observado en periodos anteriores, en esta etapa se privilegió la construcción de viviendas económicas terminadas, es decir la entrega de soluciones habitacionales completamente construidas (MINVU 2007). El subsidio cubría parte de los costos de la vivienda por lo que los beneficiarios debían endeudarse para pagar la diferencia. Esta situación refleja la predominancia de la semántica económica en la forma en que el problema de la vivienda es abordado durante este periodo. 
Las presentes Bases están destinadas a reglamentar la adquisición de "soluciones habitacionales de interés social" y "viviendas económicas" terminadas, y cuyos proyectos han sido precalificados. Esta adquisición se efectuará mediante licitación pública por los Servicios de Vivienda y Urbanización, en adelante SERVIU. (Decreto $\mathrm{N}^{\circ} 1292$. MINVU, 03 de enero de 1978)

Las personas beneficiarias de los programas a que se refiere el presente reglamento podrán solicitar un crédito hipotecario complementario para enterar el pago del precio de la vivienda, a un Banco o Sociedad Financiera, conforme a las normas del Consejo Monetario, en las condiciones siguientes. (Artículo N4, Decreto $\mathrm{N}^{\circ} 235$. MINVU, 6 de febrero de 1986)

Es importante señalar que durante este periodo, complementariamente a la referencia económica, la noción de familia actúa como un criterio diferenciador de quienes son observados como los públicos a los que el Estado dirige sus acciones. El concepto familia adquiere cada vez mayor presencia en las leyes y decretos de esta etapa, a partir de denominaciones tales como 'personas casadas', 'grupo familiar' y la estimación del número de hijos que las componen. Esto denota una concepción tradicional que define a la familia por el contrato legal que se establece entre parejas heterosexuales y la existencia de hijos, dejando fuera otras conformaciones y composiciones familiares. Como se afirma hacia 1984 cuando se establecen los criterios de postulación al subsidio:

Presentar, su Rol Único Tributario y su Cédula de Identidad y los de su cónyuge; su Libreta de Familia o el certificado de matrimonio y los certificados de nacimiento de sus hijos menores de 18 años. (Artículo $\mathrm{N}^{\circ} 7$, Decreto $\mathrm{N}^{\circ} 62$. MINVU, 20 de junio de 1984)

En base a lo anterior podemos decir que los destinatarios de la política estatal de vivienda pasan a ser aquellas familias de más bajos ingresos que son clasificadas como tales a partir de un rígido sistema de estratificación socioeconómica relativo a las posesiones de cada grupo familiar. La relevancia de esta forma de entender a la familia como objeto de la política pública se observa además en los criterios de asignación de viviendas, las que según sus dimensiones eran destinadas a grupos familiares que variaban en relación a la cantidad de miembros que los componían:

A las viviendas con tres dormitorios sólo podrán optar los postulantes que tengan cuatro o más hijos menores de 18 años; a las de dos dormitorios, postulantes con dos o más hijos menores de esa edad, y a las de un dormitorio, los postulantes en general. Sin embargo, los SERVIU, previa autorización de la Secretaría Ministerial respectiva, podrán modificar esta norma en casos calificados, debiendo señalarse esta modificación en el aviso sobre la selección a efectuarse a que se refiere el artículo $9^{\circ}$. (Artículo $\mathrm{N}^{\circ} 10$, Decreto $\mathrm{N}^{\circ} 62$. MINVU, 20 de junio de 1984) 
La preponderancia de las referencias económicas que se observan durante este periodo muestran cómo las transformaciones semánticas y operativas en la política pública y las legislaciones asociadas a ésta no implican necesariamente un aumento progresivo de complejidad. Por el contrario, el problema de la vivienda pasa a ser comprendido exclusivamente como un problema económico. La misma noción de déficit que anteriormente conjugaba una serie de referencias simbólicas, se restringe a la estimación de recursos requeridos para responder a la demanda de viviendas. Por otro lado, los públicos con los que el Estado se comunica se distinguen en función de dos criterios complementarios: familias tradicionales y carencia de recursos económicos. Las semánticas con referencias técnicas, jurídicas y normativas, que en las etapas anteriores determinaban como el Estado definía el problema de la vivienda, se ven supeditadas a la primacía de la economía en la implementación de acciones estatales para abordar las necesidades de los más carenciados. Esta situación, sin embargo, cambia radicalmente con el retorno a la democracia, hito que inaugura el último periodo de análisis. Como veremos a continuación, éste se caracteriza por la diferenciación entre diversos públicos y estrategias sobre el modo de abordar el aumento de las demandas al Estado por la solución del problema de la vivienda, lo que significa un aumento sustantivo de complejidad en relación con todos los periodos anteriores.

\section{LA SEMÁNTICA DE LA INCLUSIÓN EN LA CONSTRUCCIÓN DEL PROBLEMA DE VI-} VIENDA (1990-2010)

A partir de la década de 1990, la identificación de las necesidades particulares de diversos públicos repercutió en la configuración de una política de vivienda amplia y compleja que incorporó nuevas referencias, expectativas y operaciones. La principal característica de este periodo es la diversificación de los públicos que el Estado identifica como foco de intervención. Esto implica que las características económicas de la población dejan de ser el criterio primordial en la configuración del problema de la vivienda, a la vez que la orientación de la toma de decisiones evoluciona hacia la inclusión funcional de los destinatarios de esta política. El periodo culmina el año 2010, cuando la disposición de terrenos para la construcción de viviendas sociales se vio reducida, luego de una etapa de construcción masiva de este tipo de soluciones, lo que además repercutió en el encarecimiento de los espacios disponibles para ello. A esto se suma el hecho de que, a partir de las necesidades de dotación de viviendas de emergencia para personas afectadas por el terremoto del año 2010, la destinación de recursos y los focos de atención política cambiaron sustantivamente.

El aumento del déficit de viviendas que se generó en el periodo anterior, junto con el incremento en las cifras de pobreza (Hidalgo 2004), implicaron que la referencia económica en la definición del problema se 
mantuviera como una de las orientaciones fundamentales de la política pública. Una de las principales medidas que se toma al respecto fue la entrega de subsidios que, junto con un ahorro mínimo por parte de los postulantes, cubrían el gasto total del terreno y la construcción de la vivienda (MINVU 2007). Junto con lo anterior, se implementaron políticas de amortización de las deudas de quienes habían adquirido una vivienda en el período anterior (Sugranyes 2005). En este contexto, a las denominaciones económicas de 'pobreza' e 'indigencia', se suma la distinción de los 'deudores habitacionales' como parte de los públicos que el Estado procura atender.

Considerando: Que atendido que mediante el D.S. N5(V. y U.), de 2002, se implementó por el Ministerio de Vivienda y Urbanismo la nueva política habitacional, conforme a la cual los créditos para el financiamiento de viviendas debían obtenerlos los postulantes de instituciones financieras, se ha estimado conveniente promover la extinción o amortización de las deudas que mantienen deudores habitacionales que se encuentran en condiciones de indigencia o de pobreza, con las instituciones financieras que les hubieren otorgado dicho crédito en virtud de convenios suscritos al efecto por dichas entidades crediticias con el Ministerio de Vivienda y Urbanismo conforme al artículo 21 bis del D.S Nº2 (V. y U.), de 1984, en su texto reemplazado por D.S. N5 (V. y U.), de 2002. (Decreto N²30. MINVU, 14 de enero de 2006)

Otra característica relevante de las políticas de estos años es diversificación de los tipos de familias a los que se dirigen los recursos estatales, lo que marca una clara diferencia con el periodo precedente. Una de estas distinciones corresponde a la de familias extendidas que habitan en viviendas de limitadas dimensiones. A partir de la identificación de estas situaciones, se focalizaron recursos para atender las necesidades de que aquellas familias no propietarias que residían en viviendas de dimensiones insuficientes, fenómeno al que se denominó como 'allegamiento' (Rugiero 1998).

Los SERVIU, con cargo a sus recursos presupuestarios o a los que se pongan a su disposición para estos efectos, desarrollarán programas de viviendas progresivas, para la atención de familias que vivan allegadas o que se encuentren en otras situaciones de emergencia habitacional, sea construyendo dichas viviendas para asignarlas a sus destinatarios o concurriendo a su financiamiento. (Artículo Nº1, Decreto $\mathrm{N}^{\circ} 140$. MINVU, 22 de octubre de 1990)

Junto con lo anterior, se consideraron como familias factibles de ser beneficiarias de la política de vivienda aquellas cuyos miembros son personas solteras, viudas, madres y padres solteros, padres o madres de la pareja principal e incluso a los hijos aún no nacidos. También se incorporaron como públicos particulares a las familias indígenas, adultos mayores y familias con miembros que presentan una discapacidad. En atención a la diversidad de requerimientos de estos distintos tipos de personas, se 
incorporaron criterios de diferente naturaleza en los procesos de postulación y asignación de subsidios. En esto se observa que, ante un problema complejo, construido a partir de la diferenciación de públicos y demandas, la política responde incorporando especificaciones técnicas, económicas y culturales que buscan atender las necesidades de esta población.

Si además de tratarse de la postulación de madre o padre soltero o viudo o cuyo matrimonio ha sido declarado nulo que tenga a su cargo hijos que cumplan con los requisitos para integrar su grupo familiar, uno o más de esos hijos son menores de 15 años hasta la expiración del año calendario en el curso del cual hubieren cumplido los 15 años de edad y es acreditado como carga familiar del postulante, por esta circunstancia le corresponderán 15 puntos adicionales. Si el postulante, o su cónyuge, o uno o más miembros integrantes del grupo familiar acreditado, estuvieren inscritos en el Registro Nacional de la Discapacidad, le corresponderán 15 puntos adicionales como postulante. Si el postulante es mayor de 65 años, incluidos aquellos que los cumplan hasta la expiración del año calendario en el curso del cual hubieren cumplido los 65 años de edad, le corresponderán 15 puntos adicionales por este concepto, aunque no acrediten otro u otros miembros de su grupo familiar. (Artículo $\mathrm{N}^{\circ} 13$, Decreto $\mathrm{N}^{\circ} 117$. MINVU, 11 de julio de 2002)

Como se señaló previamente, el foco de la política se centró de manera preferente en las personas en situación de pobreza pero, a diferencia de periodos anteriores, se profundiza en el diagnóstico de dicha situación, ampliado la mirada de este problema más allá de la carencia de recursos monetarios. Las necesidades a las que se busca atender refieren a las posibilidades de participación de las personas en esferas de la sociedad, tales como el trabajo, la salud y la educación. La vivienda pasa a ser entendida por el Estado como un mecanismo que debe facilitar la inclusión social de las personas en distintos sistemas sociales. A partir de la elaboración de diagnósticos de los problemas particulares de los postulantes a los subsidios de vivienda, se procuró atender las expectativas de los beneficiarios que apuntaban a mejorar sus condiciones integrales de vida.

Para el diseño del Plan, la EGIS deberá hacer un diagnóstico de las familias que conforman el grupo, identificando los principales problemas que les afectan, considerando además la situación social que tendrán las familias en la futura localización del proyecto, en ámbitos tales como vivienda, salud, educación y trabajo. Dicho diagnóstico deberá considerar los casos vulnerables que existan en el grupo, tales como discapacitados y adultos mayores, entre otros, para quienes se deberán desarrollar acciones específicas de intervención. Además, con tal propósito, la EGIS deberá llenar la ficha de diagnóstico de cada familia postulante. (Artículo N²0, Decreto Nº174. MINVU, 9 de febrero de 2006)

Ligado con lo anterior, las referencias asociadas a especificaciones técnicas adquirieron especial relevancia en la determinación de normas de construcción de las viviendas y el establecimiento de requisitos básicos del entorno urbano en el que estas se sitúan. El acceso a servicios y vías de transporte pasan a ser exigencias para el desarrollo de proyectos destinados a 
agrupaciones de familias que postulan en forma conjunta a los subsidios habitacionales implementados en este periodo (Castillo 2010; Pincheira 2014).

Para aplicar el subsidio regulado por el presente reglamento, el proyecto habitacional deberá cumplir con las siguientes condiciones: * No exceder un tamaño máximo de 300 viviendas; * Estar ubicado dentro del límite urbano fijado por el respectivo instrumento de planificación territorial; ${ }^{*}$ Estar ubicado dentro del territorio operacional de la empresa sanitaria correspondiente; * Tener acceso directo a una vía local o de rango mayor existente; $y^{*}$ Contar con acceso a transporte público. Además, las viviendas correspondientes a estos proyectos deberán cumplir con el itemizado técnico aprobado por resolución exenta $\mathrm{N}^{\circ} 2.763$ (V. y U.), de 2005 y su programa arquitectónico deberá contar, a lo menos, con dos dormitorios conformados, baño, estar-comedor y cocina, pudiendo esta última encontrarse incorporada al estar-comedor. Excepcionalmente, en casos calificados y por razones fundadas, el Director del Serviu podrá autorizar rebajar los niveles de exigencia establecidos en este inciso. Con todo, el Ministro de Vivienda y Urbanismo podrá, en casos calificados y mediante resolución fundada, eximir de una o más de las exigencias señaladas en este artículo. (Artículo N4, Decreto $\mathrm{N}^{\circ}$ 4. MINVU, 30 de octubre de 2009)

El concepto 'proyecto habitacional' da cuenta de la relevancia que la planificación técnica de las acciones estatales adquiere progresivamente en cada una de las políticas implementadas a partir de la década de 1990. A su vez, este concepto da cuenta de la importancia asignada la postulación a soluciones habitacionales de carácter colectivo a las que es posible acceder luego de la conformación jurídica de un 'comité de vivienda'.

Cabe señalar que durante este periodo se generaron modalidades especiales de subsidios habitacionales dirigidos a personas y familias no pobres. El subsidio de renovación urbana y las normas de sobre arrendamiento de vivienda con promesas de compraventa, son innovaciones que se incorporaron al sistema unificado de subsidio habitacional (Simian 2010). A partir de ello, el Estado implementa acciones destinadas a los sectores medios como parte de los públicos con que se relaciona. Este es otro ejemplo de la mayor diversidad de situaciones y expectativas que se integran durante este periodo:

Autorízase a los bancos, sociedades financieras y cajas de compensación de asignación familiar, en adelante, "las instituciones", para abrir y mantener cuentas de ahorro para el arrendamiento de viviendas con promesa de compraventa, de acuerdo con las normas de esta ley, con objeto de recibir en ellas el ahorro metódico y el ahorro voluntario que efectúen sus titulares, a fin de pagarlas rentas de arrendamiento y acumular fondos suficientes para financiar la compra de las viviendas arrendadas en el plazo convenido entre las partes. Los titulares de estas cuentas sólo podrán ser personas naturales. (Artículo N¹, Ley N¹928. MINVU, 27 de diciembre de 1993) 
En síntesis, lo que predomina durante este periodo es la vinculación de expectativas que se generan a partir de la identificación de diversos tipos de públicos. La incorporación de necesidades particulares de personas diferentes, la ampliación de la concepción de familia, la importancia que se le otorga al acceso a diversos servicios sociales, todo esto da cuenta de un giro relevante de la política de vivienda. La definición del problema se amplía a una noción compleja que procura integrar diversidad de posiciones individuales y la diversidad de necesidades funcionales de participación en la sociedad. Se entiende que, para que esto sea efectivo, es necesario generar oportunidades de participación en distintas esferas sociales y que la inclusión en cada una de ellas estará relacionada con la inclusión en las demás.

\section{CONCLUSIONES}

A partir del caso de la política de vivienda en Chile hemos ilustrado cómo la política pública define los problemas a los que dirige sus operaciones y rendimientos. Para ello, nos hemos centrado en el análisis de la evolución de las formulaciones semánticas que el sistema político elabora a partir de los contenidos comunicativos expresados en las demandas de actores sociales. Observamos cómo la semántica política se construye heterorreferencialmente a partir de orientaciones simbólicas que provienen de distintos sistemas, las que influyen en la forma en la que el problema es entendido y abordado por el Estado. Junto con esto, hemos evidenciado cómo estas referencias simbólicas cambian a través del tiempo a partir de variaciones de las necesidades y demandas de los actores sociales.

El problema de la vivienda se configura inicialmente como un problema de salud pública, referencia que es reemplazada por semánticas de carácter técnico, económicas, jurídicas, asociadas al entorno urbano y la inclusión. Tales referencias oscilan en el nivel de influencia que tienen sobre la definición del problema en distintos períodos de la política de vivienda. Cabe destacar que los cambios observados no demarcan un trazado lineal de aumento de complejidad. Esto se verifica en el tercer periodo de análisis, en el que el problema se simplifica a partir de la predominancia que asumen las semánticas económicas. El análisis indica que la oscilación de la que hablamos se relaciona con la definición de cuáles son los públicos que el Estado construye internamente en función de las demandas de distintos actores sociales. En principio se trata de los obreros urbanos que han migrado a las grandes ciudades del país, para luego centrarse en las necesidades de trabajadores, personas de menores recursos, pobladores, sectores medios, distintos tipos de familias e individuos. En las leyes y decretos revisados estos públicos se operacionalizan como beneficiarios de la política de vivienda, lo que permite identificar hacia donde el Estado dirige sus operaciones. Los públicos varían en distintos periodos sin que haya un patrón continuo de cambio. Estos dependen de las distinciones que el sistema 
político realiza sobre su entorno y, por tanto, del tipo de actores con los que se vincula y se deja de vincular en distintos momentos. Lo que para el Estado resulta relevante como distinción depende de quiénes observa y no necesariamente de la cuantía de estas observaciones.

A partir de la estrategia de análisis desarrollada en este trabajo se ha trazado un recorrido histórico por las principales leyes y decretos que representan distintos tipos de acciones para abordar el problema de la vivienda. Se identificaron cuatro periodos de análisis en función de hitos que dan cuenta de cambios relevantes en la forma en como el Estado comprende e interviene el problema de la vivienda. Esto hitos son: la primera ley que aborda la temática habitacional, la centralización de la intervención a partir de la creación del MINVU, la implementación del subsidio a la vivienda como estrategia focalizada en el traspaso de recursos económico, el aumento del gasto público y la re-destinación de recursos en función del déficit de territorios para la construcción de viviendas sociales, y las necesidades generadas por el terremoto de 2010.

Los cambios operativos de las políticas públicas pueden ser interpretados como un correlato de las oscilaciones semánticas sobre la definición del problema y, en tal sentido, denotan cómo las semánticas tienen consecuencias operativas en la implementación de políticas públicas. Los cambios semánticos por los que atraviesan las políticas públicas dan cuenta de cómo el sistema político aborda operativamente y coordina las demandas de los actores de su entorno. En tal sentido, la centralización que se logra con el MINVU es una respuesta a la fragmentación que caracteriza al primer periodo, donde las decisiones se toman de manera difusa y en cierto sentido caótica por parte de las distintas instituciones del Estado. Asimismo, la implementación del subsidio a la vivienda procura simplificar y focalizar la asignación de recursos públicos como reacción a estrategias previas en las que el Estado se presentaba como el principal responsable de la intervención del problema de la vivienda y obtenía resultados moderados en relación con las expectativas de los públicos. El aumento del gasto social da cuenta de la necesidad de que el Estado retome la responsabilidad de reducir el amplio déficit de vivienda generado en el periodo anterior (MINVU 2007). Cabe señalar que, para que se produzcan estas variaciones, es fundamental que el Estado amplíe su capacidad de observación del entorno, distinguiendo e integrando las diferentes demandas que los actores sociales van presentando. Sobre la base de dicha capacidad es posible que estabilice sus operaciones durante los períodos indicados.

Con la demarcación temporal de periodos se ha puesto énfasis en los procesos internos de transformación de las políticas públicas, demostrando que estos son independientes de los periodos de gobierno. Hablamos de la evolución de la política pública como política de Estado, proceso operativo de toma de decisiones donde confluyen referentes sistémicos diversos. Es 
importante aclarar que esto no quiere decir que la política pública se reduzca solo a la esfera estatal de implementación, dado que se reconoce la relevancia e influencia que los actores privados han ido ejerciendo progresivamente en su diseño, implementación y evaluación. Lo que se pretende destacar es la lógica interna de reproducción de la política pública a partir de construcciones semánticas de los problemas que aborda.

Cabe explicar por qué el análisis se detiene el año 2010, dejando fuera lo acontecido los últimos 6 años. Dos razones justifican esta decisión, una empírica y otra metodológica. El éxito de las políticas implementadas durante la década del 2000, en relación con la disminución sustantiva del déficit de vivienda (Hidalgo 2004), implicó que los terrenos y viviendas sociales disponibles empezaran a decrecer, paralelamente al aumento de sus precios. Junto con esto, el terremoto del año 2010 significó la reasignación de recursos a la construcción de viviendas de emergencia, la reparación y construcción de nuevas viviendas (Polanco 2012), lo que implicó un cambio de foco en relación con los destinatarios de esta política. Esto evidencia variaciones en las estrategias operativas con las que el problema de la vivienda se venía abordando en este último periodo. A estos datos empíricos se suma una precaución metodológica relacionada con la incorporación de datos recientes en el análisis de información histórica. La menor disponibilidad de normativas, evaluaciones e investigaciones científicas de lo acontecido estos últimos 6 años debilitaría el estudio de este período respecto de los anteriores. Por otro lado, no se cuenta con la distancia suficiente para una observación de esta información con una perspectiva más amplia.

El análisis también muestra la necesidad de complementar el conocimiento con investigaciones futuras. Estas debieran centrarse en: a) las contribuciones que otros actores no estatales realizan a la formación de políticas públicas, para de ese modo contrastarla con la semántica estatal revisada en este artículo; b) en el mismo sentido, también es relevante observar la construcción semántica de los públicos de la política y su sintonía o distancia con la construcción semántica del Estado. Triangular estas fuentes de producción semántica permitiría tener un panorama completo de las intersecciones, conflictos y posibles crisis que se construyen en el despliegue de la política de vivienda en el siglo XX en Chile.

A pesar de lo anterior, el estudio de la semántica estatal permite complementar de manera fructífera el análisis de las políticas públicas basado en el concepto de coordinación social. Como observamos, en la semántica de la política pública confluyen orientaciones simbólicas de distintos sistemas parciales, esto significa que la política condensa esta red de significados en lo que define como problema. Esto puede ser interpretado como coordinación en tanto se trata de la vinculación de lógicas sistémicas. Se puede sostener que los intereses de los actores involucrados en la construcción de las políticas públicas expresan estas orientaciones sistémicas y, por lo tanto, se asocian a esta red de referencias semánticas, a 
partir de las cuales la política pública construye sus operaciones. Esto se observa en el caso de la política de vivienda, donde son intereses económicos los que motivan a los actores privados a involucrarse en la construcción de viviendas sociales, así como los centros de investigación y sus expertos desarrollan aportes asociados a sus intereses científicos de generación de conocimiento sobre el tema.

El enfoque empleado pretende ser un aporte al estudio y evaluación de las políticas públicas en tanto: a) da cuenta de los procesos internos de desarrollo de las políticas públicas y comprende con ello cómo estas evolucionan; b) devela cómo el Estado construye los públicos a los que dirige sus acciones; c) da cuenta de cómo la semántica política integra referencias simbólicas de los distintos sistemas con los que se vincula en la observación de problemas sociales. En este conjunto de herramientas se encuentra directamente relacionado con contribuciones y definiciones de carácter teórico, lo que robustece la capacidad de interpretación de esta propuesta. Sobre la base de estos fundamentos conceptuales es posible comprender la evolución de las operaciones del Estado en relación con procesos de transformación y complejización de la sociedad. Esto representa una ventaja comparativa en relación con otros enfoques, si consideramos que gran parte de las evaluaciones de las políticas públicas se basan en criterios técnicos de análisis de resultados e impactos que son entendidos como procesos lineales de causas y efectos de limitado alcance explicativo.RM

\section{REFERENCIAS}

Arnold, M. (2012). El debate sobre las desigualdades contemporáneas: ¿puede excluirse la exclusión social? Revista Mad, 27, 34-43.

Arellano, J. P. (1982). Políticas de Vivienda Popular: Lecciones de la experiencia Chilena. Santiago de Chile: Coleccion Estudios CIEPLAN Nº 9.

Atkinson, A. (2009). The EU and Social Inclusion: Facing the Challenges. Bristol: Policy Press.

Börzel, T. \& Risse, T. (2010). Governance without a State: Can it Work?. Regulation \& Governance, 4(2), 113-134.

Cadenas, H. (2016). La desigualdad de la sociedad. Diferenciación y desigualdad en la sociedad moderna. Persona y Sociedad, 26(2), 51-77.

Castillo, M. (2010). Producción y gestión habitacional de los pobladores. Participación desde abajo en la construcción de vivienda y barrio en Chile. Cuadernos Electrónicos de Derechos Humanos y Democracia, 6, 30-71.

De Ramón, A. (2000). Santiago de Chile (1541-1991). Historia de una sociedad urbana. Santiago: Sudamericana.

Dockendorff, C. (2007). Teoría sociológica, cultura moderna y emancipación: Un ejercicio inconcluso de auto-aclaración sociológica. Revista Mad, 16, 1-17.

Ducci, M. (1997). Chile: el lado oscuro de una política de vivienda exitosa. EURE, 23(69), 99-115. 
Forrary, R. (2007). Participación: compartir los poderes de decisión y acción sobre la ciudad. En M. Castillo \& R. Hidalgo (Eds.), Cien años de política de vivienda en Chile (pp. 129-149). Santiago de Chile: Ediciones Universidad Católica de Chile.

Geisse, G. \& Valdivia, M. (1978). Urbanización e industrialización en Chile. EURE, 5(15), 11-35.

Habermas, J. (1973). Legitimation Crisis. Cambridge: Polity Press.

Hidalgo, R. (1999). La vivienda social en Chile: la acción del estado en un siglo de planes y programas. Scripta Nova, 45.1

Hidalgo, R. (2004). La vivienda social en Santiago de Chile en la segunda mitad del siglo XX: Actores relevantes y tendencias espaciales. En C. De Mattos C., Ducci, A. Rodriguez, \& G. Yáñez (Eds.). Santiago en la Globalización. ¿Una nueva ciudad? (pp. 219-242). Santiago de Chile: Ediciones Sur.

Kjaer, P. (2014). Constitutionalism in the Global Realm. Oxon: Routledge.

Lechner, N. (2003). Tres formas de coordinación social. Nueva Época, 1(2), 5-16.

Luhmann, N. (1991). Sistemas sociales. Lineamientos para una teoría general. México DF: Iberoamericana, Alianza Editorial.

Luhmann, N. (1993). Teoría política del Estado de Bienestar. Madrid: Alianza.

Luhmann, N. (2007). La sociedad de la sociedad. México DF: Herder.

Luhmann, N. (2009). La política como sistema. México DF: Universidad Iberoamericana.

Mayntz, R. (2001). El estado y la sociedad civil en la gobernanza moderna. Reforma y democracia, 21, 7-22.

Mascareño, A. (2010). Coordinación social mediante políticas públicas: el caso chileno. Revista de la CEPAL. 101, 111-126.

Mascareño, A., \& Carvajal, F. (2015). Los distintos rostros de la inclusión y la exclusión. Revista CEPAL. 116, 131-146

MINVU. (2007). Un siglo de políticas en vivienda y barrio. Santiago de Chile: Peguen.

MINVU. (2004). Política de la Vivienda en Chile: Discurso del Ministro de Obras Públicas D. Modesto Collao. Santiago de Chile: MINVU.

MINVU. (1964). Política de la Vivienda en Chile. Discurso del Ministro de Obras Públicas D. Modesto Collados. Santiago de Chile: MINVIU.

Parsons, T. (2007). American Society. A Theory of the Societal Community. Boulder: Paradigm Publishers.

Pincheira, V. (2014). Políticas habitacionales y la falta del derecho a la vivienda en Chile. Revista de Derechos Fundamentales. 11, 57-92.

Polanco, M. (2012). Efectos sociales del terremoto en chile y gestión política de la reconstrucción durante el gobierno de Sebastián Piñera (2010-2011). Revista Enfoques, 10(16), 19-46.

Rawls, John, 2002. La justicia como equidad. Una reformulación. Barcelona: Paidós.

Rivera, A. (2012). Historia de la política habitacional en el área metropolitana de Santiago. Revista CIS, 16(16), 27-44.

Robles, F. (2005). Contramodernidad y desigualdad social: Individualización e individuación, inclusión/exclusión y construcción de identidad. La necesidad de una sociología de la exclusión. Revista Mad, 12, 27-57.

Rosanvallon, P. (2000), The New Social Question. Rethinking the Welfare State. New Jersey: Princeton University Press.

Rugiero, A. (2011). Experiencia chilena en vivienda social: 1980-1995. Revista INVI, 13(35), 3-87.

Sabatini, F., \& Soler, F. (1995). Paradoja de la planificación urbana en Chile. EURE, 21(62), 61-73.

Stäheli, U. (1997). Exorcising the 'Popular' Seriously: Luhmann's Concept of Semantics. International Review of Sociology, 7(1), 127-145. 
Scharpf, F. (1999). Governing in Europe: Effective and Democratic? Oxford: Oxford University Press.

Schmidt, V. (2013). Democracy and Legitimacy in the European Union Revisited: Input, Output and 'Throughput'. Political Studies, 61(1), 2-22.

Simian, J. (2010). Logros y desafíos de la política habitacional en Chile. Estudios Públicos, 117, 269-322

Sugranyes, A. (2005). La política habitacional en Chile, 1980-2000: un éxito liberal para dar techo a los pobres. En R. Alfredo, \& A. Sugranyes. Los con techo. Un desafío para la política de vivienda social (pp. 23-57). Santiago de Chile: Ediciones SUR.

Torres, I. (1986). Los conventillos en Santiago (1900-1930). Cuadernos de Historia, 6, 67-85.

Vega, O. (2006). La paradoja de la política social y el Programa Puente: Superación de la extrema pobreza o inclusión/exclusión social. Revista Mad, 15, 93-103.

von Beyme, K. (1994) Teoría política del siglo XX. De la modernidad a la postmodernidad. Madrid: Alianza.

Willke, H. (2007). Smart Governance. Governing the Global Knowledge Society. Frankfurt aM, New York: Campus.

Willke, H. (2014). Concepciones de regulación política y problemas de gobierno político. Economía y Política, 1(1), 75-118.

Zeitlin, J., Pochet, P., \& Magnusson, L. (2005). The Open Method of Co-ordination in Action: The European Employment and Social Inclusion Strategies. Brussel: P.I.E.Peter Lang.

Ziccardi, A. (2008). Pobreza urbana y políticas de inclusión social en las comunidades complejas. Bitácora Urbano Territorial, 2(13), 93.

\section{SOBRE LA AUTORA}

Gabriela Azócar (Doctora en Sociología) es Investigadora Adjunta del Centro de Investigación Núcleo Milenio Modelos de Crisis (NS 130017) y Profesora del Departamento de Sociología de la Universidad de Chile. Sus áreas de investigación incluyen temas de políticas públicas, vivienda, metodología de investigación sistémica e intervención social. Entre sus últimas publicaciones destacan: La observación de las políticas públicas como espacios de vinculación de complejidad (En: A. Peroni \& K. Dominique (Eds.), Investigación Evaluativa de Políticas Públicas, 2013), Hacia la delimitación de un marco teórico conceptual para la investigación evaluativa (Revista Mad, 30, 2014).

\section{CONTACTO}

Centro de Investigación Núcleo Milenio Modelos de Crisis

Escuela de Gobierno

Universidad Adolfo Ibáñez

Diagonal Las Torres 2640

Santiago, Chile

CP: 7941169

gazocarde@uchile.cl

Recibido: marzo 2016

Aceptado: julio 2016 